\title{
The applicability of mathematics to physical modality
}

\author{
Nora Berenstain ${ }^{1}$
}

Received: 31 July 2015 / Accepted: 26 February 2016

(C) Springer Science+Business Media Dordrecht 2016

\begin{abstract}
This paper argues that scientific realism commits us to a metaphysical determination relation between the mathematical entities that are indispensible to scientific explanation and the modal structure of the empirical phenomena those entities explain. The argument presupposes that scientific realism commits us to the indispensability argument. The view presented here is that the indispensability of mathematics commits us not only to the existence of mathematical structures and entities but to a metaphysical determination relation between those entities and the modal structure of the physical world. The no-miracles argument is the primary motivation for scientific realism. It is a presupposition of this argument that unobservable entities are explanatory only when they determine the empirical phenomena they explain. I argue that mathematical entities should also be seen as explanatory only when they determine the empirical facts they explain, namely, the modal structure of the physical world. Thus, scientific realism commits us to a metaphysical determination relation between mathematics and physical modality that has not been previously recognized. The requirement to account for the metaphysical dependence of modal physical structure on mathematics limits the class of acceptable solutions to the applicability problem that are available to the scientific realist.
\end{abstract}

Keywords Scientific realism - Indispensability argument - Applicability problem . Modality $\cdot$ Mathematical explanation $\cdot$ Novel prediction

Nora Berenstain

nberenst@utk.edu

1 University of Tennessee, Knoxville, USA

Published online: 24 March 2016 


\section{Introduction}

How can it be that mathematics, the study of what is often taken to be an abstract realm of structures and relations, is so relevant to our study of the concrete physical world? Scientists not only model the empirical world mathematically, they use mathematical structures to make inferences and novel predictions about it. Wigner (1960) refers to "the unreasonable effectiveness of mathematics in the natural sciences." What could explain the astonishing success of applying mathematics to physical systems? This question is known as the applicability problem. Initially re-popularized by Steiner (1978, 1989, 1995, 1998), it has been more recently addressed by Colyvan (2001a,b), Pincock (2004a, 2007), Bueno and French (2012), Ye (2010), and Bangu (2012).

This paper explores the connections among the applicability problem, the nomiracles argument for scientific realism, and the indispensability argument for mathematical realism. ${ }^{1}$ The goal is to show that certain features of the indispensability argument for mathematical realism limit the set of possible solutions to the applicability problem that are available to the scientific realist. In Sect. 2, I identify an analogy between the no-miracles argument for scientific realism and the indispensability argument for mathematical realism that suggests a restriction on possible answers to the applicability problem. I suggest that there is an important parallel between the role of unobservable entities and the role of mathematical structures in science. Just as unobservable entities are taken to explain the behavior of observable entities when they cause or otherwise nomically necessitate such behavior, mathematical structures cannot be explanatory unless they bear some determination relation to the observable structures they are taken to explain.

I argue that this insight must be taken to limit the class of possible solutions to the applicability problem that are available to the scientific realist. I conclude that the scientific realist must posit a relation of metaphysical dependence between mathematical structure and modal physical structure. In Sect. 3, I discuss commonly cited instances of mathematical explanation in the sciences in order to show that the explananda are modal in each case. I also introduce a case in which mathematical structure was indispensable to the novel prediction of an empirical phenomenon. In Sect. 4, I discuss how these cases bear on ways that the scientific realist can answer the applicability problem and I show that the primary purported solution offered by the mapping account is unsatisfactory. This argument forecloses on solutions to the applicability problem that do not posit a relation of metaphysical dependence between mathematical structure and modal physical structure and limits the class of solutions that the scientific realist can accept to those that do.

\footnotetext{
1 The conclusions of the indispensability argument remain disputed. For the purposes of this paper, it is assumed that the indispensability argument is sound. As the indispensability argument has scientific realists as its audience, scientific realism is also assumed here. For background on the connection between indispensability and scientific realism, see Colyvan (1999, 2001a, b, 2006). Broadly, the indispensability argument can be seen as an extension of the no-miracles argument to mathematical entities, as indispensability theorists take there to be no in-principle difference between the role that mathematical entities play in theoretical explanations of observable phenomena and the role that non-mathematical theoretical entities play in such explanations.
} 
Section 5 addresses potential relations for the type of metaphysical dependence that holds between mathematical and modal physical structure. These include Grounding, supervenience, instantiation, and identity. I suggest that the instantiation of mathematical structures by modal physical structures is the most promising candidate for the relevant type of metaphysical dependence.

\section{Scientific realism and the indispensability argument}

According to the no-miracles argument, scientific realism is the only view that does not make the success of science a miracle. These successes include science's ability to make novel predictions, offer explanations, and unify seemingly disparate phenomena. Since many novel predictions and scientific explanations of observable phenomena make reference to unobservable entities, we are committed to the existence of unobservables as metaphysically real entities beyond mere theoretical constructs. ${ }^{2}$ The no-miracles argument for scientific realism commits us not only to unobservable entities but to a necessary connection between the unobservables and the observable phenomena they are posited to explain. ${ }^{3}$ This necessary connection is frequently though certainly not always taken to be a causal one. ${ }^{4}$ I suggest that, just as in the case for scientific realism, the argument for realism about mathematical entities commits us not only to the existence of the entities themselves but to a metaphysical determination relation between the entities and the empirical phenomena they predict and explain. In the next section, I show that the empirical phenomena that mathematical structures predict and explain are inherently modal. Thus, the behaviors and properties of modal physical systems can be explained and predicted by mathematical structures and properties when the former are grounded in the latter. ${ }^{5}$

Consider Baker's (2009) enhanced indispensability argument for mathematical realism:

1. We ought to have ontological commitment to all and only those entities that are explanatorily indispensable to our best scientific theories.

2. Mathematical entities are explanatorily indispensable to our best scientific theories.

3. Therefore, we ought to be ontologically committed to mathematical entities.

The first premise is motivated by Quinean naturalism, the view that there are no nonscientific standards that we can use to assess our ontological commitments. The second is supported by cases such as those discussed in Sect. 3. In order for these cases to

\footnotetext{
2 The version of scientific realism assumed here is a metaphysical one, though the conclusions of the paper are compatible with both standard entity realism and ontic structural realism.

${ }^{3}$ See Berenstain and Ladyman (2012) for an argument that scientific realists must be committed to a non-Humean understanding of causation and laws of nature.

${ }^{4}$ For what it's worth, I take there to be numerous examples of both casual and non-causal relations holding between unobservable entities and the observable phenomena they explain. See Berenstain (2016) for further discussion.

5 The term 'grounding' as used here denotes the broad family of relations of metaphysical dependence. It is not meant to invoke the primitive relation that Wilson (2014) refers to as "Big-G Grounding," nor does it imply anything about what specific relation of metaphysical dependence must hold between modal and mathematical structures.
} 
provide substantive support for premise 2 , it must be the case that the mathematics in each case is truly explanatorily indispensable rather than merely being explanatorily helpful or representationally indispensable.

Whether the cases described are ones in which the mathematics is genuinely explanatorily indispensable remains contested. It has been disputed by Melia (2000), Leng (2002), Pincock (2004a), Saatsi (2011), and Rizza (2011), and defended by Lyon and Colyvan (2008), Baker (2009), Colyvan (2010), and Lyon (2012). I do not explicitly offer a defense of premise 2 here, though I do elucidate the specific explanatory indispensability at evidence in each of the cases discussed.

Before turning to the supporting examples, let me add a further claim. Mathematical entities are indispensable for the prediction and explanation of modal facts, as Lyon (2012) notes. ${ }^{6}$ The explanations into which they figure are explanations of possible and necessary features of empirical systems. Thus, the empirical explanations in which mathematical entities play an indispensable role should generally be recognized as having modal explananda.

\section{Mathematical structure, novel prediction, and scientific explanation}

What follows are four examples demonstrating the indispensability of mathematics to the sciences. The first is an example of mathematics being indispensable to the novel prediction of an empirical fact; the other three are examples of indispensably mathematical explanations of empirical phenomena. In the discussion following, I show that the phenomenon in each case is a modal one. I invoke the notion of modal physical structure to capture the inherently modal features and relations of the empirical entities and systems discussed. Broadly, a modal physical structure is a web of relations of nomological necessity that hold among the various entities and properties that form a physical system or phenomenon. The concept of modal physical structure is compatible with ontic structural realism, though that view is not assumed here; a non-Humean conception of modality that acknowledges natural necessity, however, is presupposed. For present purposes, nothing especially substantive need be assumed about the notion of structure in the modal physical case or in the mathematical one.

\subsection{The Glashow-Weinberg-Salam model}

In 1979, Steven Weinberg, Sheldon Lee Glashow, and Abdus Salam received the Nobel Prize in physics for their unification of the electromagnetic force and the weak force. In their search for an underlying mathematical structure that could unify the two forces, the physicists postulated that the weak and electromagnetic interactions were both governed by some group of exact local gauge symmetries, that this group was spontaneously broken to U(1) giving mass to all vector bosons except the photon, and that the theory was renormalizable (Weinberg 1979). They hypothesized that the relations between the two forces and their carrier particles were embedded within the

\footnotetext{
6 This bears emphasizing, as not all scientific realists embrace the commitment to a robust conception of modality that scientific realism seems to require. See, for example, Psillos (2002).
} 
$\mathrm{SU}(2) \times \mathrm{U}(1)$ gauge group. The resulting electroweak theory postulated the $\mathrm{W}$ boson necessary to explain beta decay-what they were originally seeking to explain-as well as the previously unobserved and unknown $\mathrm{Z}$ boson. The embedding of the known and hypothesized bosons within the group structure matched the known properties of the photon and the postulated properties of the $\mathrm{W}$ boson, which was the particle they were attempting to account for in the model. But it also left room for an additional unexpected particle, a spin-zero boson with neutral charge-what is now known as the $\mathrm{Z}$ boson. In 1983, experiments at CERN confirmed the existence of the $\mathrm{Z}$ boson. The unified electroweak theory successfully predicted the existence of an unexpected neutral fundamental particle that, along with the electrically charged $\mathrm{W}$ boson, carries the weak force.

The theoretical unification of known and hypothesized particles within a mathematical framework allowed for the prediction of a novel empirical entity. The mathematical structure of the gauge group and a spontaneously broken gauge symmetry were indispensable to its discovery. Of course, there were additional substantive assumptions that went into the creation of the model and thus allowed the gauge group to make the predictions it did. For instance, it was necessary to hypothesize some mechanism for the spontaneous breaking of $S U(2) \times U(1)$. Weinberg identified the only kind of field in the renormalizable group that could give mass to the electron and assumed that those were the only scalar fields in the theory for the sake of simplicity. But after such choices were established the predictive power of the mathematical structure of the specific gauge group could be expressed. According to Weinberg, "Once one decides on the menu of fields in the theory, all details of the theory are completely determined by symmetry principles and renormalizability, with just a few free parameters, ${ }^{7}$, (1979, p. 549). The symmetry principles of the $\mathrm{SU}(2) \times \mathrm{U}(1)$ gauge group played an essential role in establishing the theoretical content and empirically testable predictions of the unified electroweak theory.

The scientific realist should treat this case as an example of a mathematical structure being indispensable to the novel prediction of a particle. The conclusion that should be drawn is that scientific realists are committed to the entity or structure (the gauge group, in this case) that allowed for the prediction of the collection of gauge bosons. Scientific realism commits us to the view that these particles are real entities with independent existence, just as the realist takes particles to be in other cases. It would be incongruous for the realist to assert the robust independent existence of unobservable theoretical entities when their novel prediction is based in something other than mathematical structure but to take the $\mathrm{Z}$ boson's existence to be merely a feature of the theoretical formalism and thus unsurprising. If the scientific realist wants to take the novel and surprising prediction of the $\mathrm{Z}$ boson to be importantly different than standard instances of theories making novel predictions of other particles—and to thus justify not being ontologically committed to the mathematical structure that was indispensable to its prediction - then the onus is on them to offer a principled characterization of the difference.

\footnotetext{
7 These free parameters include the lepton charge and masses, the Fermi coupling constant of beta decay, the mixing angle $\theta$, and the mass of the scalar particle.
} 
It should be emphasized that the properties of the gauge bosons constitute what I have called a modal physical structure. The laws that govern their behavior describe their ranges of possible interactions with respect to the forces they carry. The particles' natures are characterized at least in part by these modal profiles. ${ }^{8}$

\subsection{Hexagonal tiling and the honeycomb theorem}

Lyon and Colyvan (2008) discuss the role that the honeycomb proof in geometry plays in explaining the hexagonal structure of hive-bee honeycomb. Consider the question of why hive-bee honeycomb is always divided up into hexagons as opposed to some other regular polygon. Their answer is two-fold:

1. Selection favors bees that minimize the amount of wax they use to build their combs over bees that use more energy than necessary by building combs with excessive amounts of wax.

2. Any partition of the plane into regions of equal area has a perimeter greater than that of the regular hexagonal honeycomb tiling.

Part (1) of the explanation makes obvious evolutionary sense. Given that scarce finite resources place limitations on survival, natural selection favors efficiency over inefficiency. Organisms that use more energy than necessary to complete a task will be less fit than conspecifics that use only what they need. Bees who produce more wax than necessary do so at the cost of not being able to complete other tasks required for the survival and reproduction of the germ-line. Darwin explains it thus, "That motive power of the process of natural selection having been economy of wax; that individual swarm that wasted least honey in the secretion of wax . . .succeeded best" (Hales 2001). Selection favors hives whose wax production is the minimum required for honeycomb production. This part of the explanation is clearly empirical.

Part (2) of the explanation, on the other hand, is purely mathematical. The theorem states that the hexagon is the polygon that tessellates a plane with the smallest resulting perimeter. As a theorem of geometry this is neither a contingent fact nor an exclusively biological one. Rather, it is a necessary fact about possible partitions of the plane into regions of equal area. No such partitions can have a total perimeter less than that of the hexagonal partition.

Suppose multiple bee populations arise in similar geographical areas with similar access to resources. One bee population uses triangles (or an approximation of them) in their construction of honeycomb, and one uses hexagons. Ceteris paribus, bee populations that use hexagonal tiling will be better off than bees that use square or triangular tiling to create their honeycombs. Given natural selection, the equilibrium state is one in which hive bees produce hexagonal honeycomb.

Let us consider what nomological factors are at play in the selection of the bees that uses hexagons over the bees that use triangles. It is purely accidental that bees ever started using hexagons in the construction of their honeycomb; to suggest otherwise

\footnotetext{
8 Berenstain (2016) offers a view on how to understand the inherently modal nature of physical properties such as being a $\mathrm{Z}$ boson. The view differs from dispositional essentialism, though it shares many of its motivations.
} 
would be to paint evolution as a forward-looking teleological force. So it is certainly not the case that it is necessary that bees produce honeycomb that is hexagonally structured. But that does not mean that there is no necessity at play in determining the structure of hive-bee honeycomb. The necessity looks something like this: If some population of bees starts using hexagons (or some approximation of them) in the production of honeycomb, all else equal, that bee population will be evolutionarily favored over a population that uses (an approximation of) some other regular polygon in honeycomb production.

That bees structure their honeycomb hexagonally is causally dependent on numerous historical and ecological contingencies: That the perimeter of their honeycombs is made by wax that they produce; that some bee population eventually stumbled upon an approximately hexagonal structure when constructing their honeycomb; that pesticides or climate change did not wipe out bee populations before they stumbled upon such a shape, etc. Though contingent on certain historical and ecological circumstances arising, that hive-bee honeycomb has a hexagonal structure is not a completely contingent fact. ${ }^{9}$ There are various necessities at play in determining that bees that produce hexagonally structured honeycomb win out. One of these necessities is the nomological one that selection in environments with scarce finite resources favors efficiency over inefficiency. Another necessity is the mathematical theorem that a hexagonal tessellation is the most efficient partition of the plane in that it maximizes area while minimizing perimeter. The mathematical necessity of this result plays an indispensible role in the explanation of the biological fact that the equilibrium structure of hive-bee honeycomb is hexagonal.

The explanandum in the explanation stated above is a modal one. To illustrate this, consider how Sober (1983) sketches the difference between causal and equilibrium explanation. He writes, "Where causal explanation shows how the event to be explained was in fact produced, equilibrium explanation shows how the event would have occurred regardless of which of a variety of causal scenarios actually transpired" (1983, p. 202). Sober's language is too strong to perfectly capture the difference between a merely causal explanation of hexagonal honeycomb and an equilibrium explanation. It is not the case that the equilibrium explanation shows how bees would have landed on hexagons regardless of what causal scenarios transpired. But he does capture the fact that the important difference between causal and equilibrium explanation is one of modal information. A merely causal explanation of the hexagonal structure of honeycomb would leave out two things: (1) the modal status of the explanandum - that the resulting phenomenon was the most likely one, all things equal, given certain conditions, and (2) the factors that are responsible for its status as equilibrium outcome and thus its increased likelihood-in this case, the geometric

\footnotetext{
9 It is helpful to distinguish between something's being contingent on certain conditions holding and its being metaphysically contingent. The two are not the same. Consider, for instance, that the modal status of ceteris paribus law can be either necessary or contingent. That a law of nature is ceteris paribus does not thereby mean it is not necessary. A law's being contingent upon certain conditions is not the same as the law's being metaphysically contingent. For if the law holds in every world in which the conditions obtain then the law, though ceteris paribus, is metaphysically necessary. See Berenstain (2014) for examples and further discussion.
} 
theorem ensuring that the hexagonal tiling is the most efficient regular tiling of the plane.

Lyon (2012) emphasizes the importance of modality in his analysis of what would be left out by a merely causal explanation that chronologically cited the different polygons bees tried out over their contingent evolutionary history. "Such an explanation (if it were actually produced) would miss a very important fact: the actual sequence of shapes tried out by the bees is irrelevant to the final outcome. So long as the bees try out hexagons at some point, no matter what other shapes the bees try, the hexagon bees win out. The mathematical explanation for why honeycombs are hexagonal gives us this important modal information." An explanation of the evolution of the hexagonal honeycomb structure that cites only the contingent causal history of the bee populations that actually produced hexagonally structured honeycomb would be incomplete. A complete explanation of why hive-bee honeycomb is hexagonally structured must incorporate the superior efficiency of the hexagon structure given scarce finite resources. The optimality of the hexagonal tiling is part of the explanation of why hive-bee honeycomb takes this structure, and its optimality is explained, indeed proved, by the geometric theorem. The mathematics in this case is indispensable to a complete explanation of the empirical phenomenon.

\subsection{Squaring the circle}

Consider now the challenge of squaring the circle, i.e., of drawing a square that has the same area as a circle in a finite number of steps using only a compass and straightedge. It cannot be done. It is a modal fact about our world that it is not possible to square the circle. What explains this fact?

The formula for the area of a circle is $\pi r^{2}$. Since $\pi$ is a transcendental number, squaring the circle involves generating a transcendental ratio, namely $1 / \sqrt{\pi}$. But only algebraic ratios can be constructed with just a compass and straightedge, namely those constructed from the integers with a finite sequence of operations of addition, subtraction, multiplication, division, and square roots. The explanation of why the circle cannot be squared is that doing so would require constructing a transcendental ratio, and only algebraic ratios can be constructed from compass and straightedge alone.

What is interesting about the case of squaring the circle is that the mathematical necessity is playing the role of prohibiting rather than ensuring certain features of physical systems. The necessity is also fairly straightforward as is the fact that it derives from mathematics. Our inability to square the circle does not come from any practical difficulty like a lack of precision in our tools. It cannot be overcome, for instance, by improvements in the technology and accuracy of compasses.

While this example is a simple one, its simplicity illustrates how easily we can construct examples of empirical phenomena whose existence is forbidden by mathematics. It is equally straightforward to create similar examples using graph theory, ${ }^{10}$

\footnotetext{
10 What Lyon (2012) did for the Bridges of Königsberg problem can also be easily done for the three-utilities problem.
} 
knot theory, or theorems of circle and sphere packing. While they may not count as instances of mathematical explanations in science, these cases are nonetheless illuminating as they are instances of empirical systems whose physical features are limited by mathematical constraints. The limitations are obviously modal. As Lyon (2012) points out, a causal explanation of why no one has ever taken a single walk that has allowed them to cross all seven bridges of Königsberg exactly once could simply cite all the walks that people have taken over the bridges and show that none of the walks have the above-stated feature. Thus a merely causal explanation would in some sense explain why it hasn't been done but it would not explain why it couldn't be done. On the other hand, a graph-theoretic explanation of the structure of the bridges explains why no one could ever take such a walk. The mathematical explanation is of a modal fact, and in this case it is a necessary one.

Examples of empirical phenomena whose necessity or impossibility are explained by theorems in mathematics are ubiquitous. While the examples of the hexagonal honeycomb in Sect. 3.2 and prime-numbered cicada life cycles in Sect. 3.4 are endlessly discussed in the literature, there is nothing particularly special about the way the mathematics operates in their explanations. We already accept that mathematical facts can place constraints on physical states of affairs in a number of trivial cases. The explanation of how this can be need not be any different or more complex in the complicated scientific cases where mathematical constraints operate alongside other modal limitations.

\subsection{Prime numbers and cicada life cycles}

Baker's (2005) example concerns the prime-numbered life cycles of the NorthAmerican Magicicada. The seven different species of this genus emerge en masse every 13 or 17 years, depending on the species. The cicadas live for 2-3 weeks, mate, and die. The nymphs then remain in the ground for the duration of the period until their next scheduled emergence.

That the emergence of adults is synchronized among all members of a cicada species in a given area has an accepted two-part explanation. Given the short lifespan of adults, fixed periodic emergence maximizes mating opportunities. Co-emergence in large numbers also leads to predator satiation, which increases the probability of individual cicada survival. What is not immediately obvious however is why the cicadas have 13- and 17-year life cycles. Two hypotheses have been proposed, and each relies on the explanatory power of the number-theoretic features of primeness.

The first proposed explanation is that long prime periods minimize intersection with periodic predators (Goles et al. 2001). Suppose that a nearby predator species had a 4-year cycle period. ${ }^{11}$ The emergence of 13 -year cicadas would overlap with the predator species' emergence only once every 4 cycles. Contrast this with a 6-year cicada species, whose emergence would coincide with the predators' every two cycles, and it is easy to see the advantage that higher prime-numbered cycle periods carry with

\footnotetext{
11 The predator hypothesis covers parasitoids that maybe have attacked adults or eggs during the period of evolutionary development but that have since become extinct (Lloyd and Dybas 1966).
} 
them. Long prime periods also minimize the chances of predator populations syncing their generations with divisors of the cicada period and coordinating population boosts in intersecting years. Thus, the predator hypothesis posits an evolutionary advantage of prime-numbered life cycles against even non-periodic predators.

The second proposed explanation appeals to another application of the numbertheoretic properties of primeness. Prime periods also minimize interaction between local cicada species with different cycle periods. This has the benefit of preventing the creation of offspring with aberrant life cycles. Suppose there were two species of cicada that had 10- and 15-year cycle periods, respectively. If members of the two species were to mate with one another and produce offspring with either a 12- or 13-year life cycle, the offspring would miss the emergence of both of the original species and thus be deprived of the opportunity to mate and reproduce (Baker 2005). Their resulting low population numbers would also mean that they fail to receive the protection offered by predator satiation.

Both proposed explanations appeal to the irreducibly mathematical features of prime numbers. Prime periods are able to minimize intersection with other periodic species because prime numbers have only two divisors. Thus, having a prime-numbered cycle period bestows an evolutionary advantage that having a composite-numbered one does not. The hypotheses obtain their explanatory power by recourse to the relevant mathematical features of the biological system's structure.

As in the honeycomb case, the mathematical facts appealed to explain a modal physical fact. Life cycles that are 13- or 17-years long are advantageous because they cannot be intersected by periods with lengths other than one or themselves. The evolutionary benefit of such long prime periods derives from this number-theoretic fact.

As in the honeycomb case, there are also a number of historical contingencies that play a role in leading up to the state of affairs we observe today. That lengthier cycle periods were selected for is explained by the evolutionary advantage of avoiding freezing summers during the glacial age, for instance. So it is not the case that the entirety of the explanation for species of the Magicicada genus evolving 13- and 17-year life cycles is mathematical. But that is not what is required for this case to demonstrate the indispensability of mathematics to the explanation of an empirical fact. When coupled with the biological fact that having a life-cycle period that minimizes intersection with nearby periodic populations is evolutionarily advantageous, facts about primeness determine the likelihood that cicada populations living near other periodic species will evolve cycle periods that are prime.

\section{Why is mathematics so applicable?}

The examples discussed in Sect. 3 demonstrate that mathematical structures and relations are indispensable to our scientific theories and explanations robustly modal features of the physical world. But even after we posit the mathematical entities required by the indispensability argument, a major question still remains: Why should mathematics be so applicable and indispensable to science? 
As Colyvan (2001b) argues, this is a question that all philosophies of mathematics face. Mathematical realism does not avoid it, nor does it answer it on its own. The conclusion of the indispensability argument, that we ought to be committed to mathematical entities, does not tell us how or why such entities figure so importantly in our explanations and predictions of the physical world. The mere existence of mathematical entities does not explain their applicability to empirical phenomena.

Pincock (2004b) introduces the mapping account, which he purports to be a solution to the applicability problem. Bueno and Colyvan (2011) characterize the account as follows, "The existence of an appropriate mapping from a mathematical structure to a physical structure is sufficient to fully explain the particular application of the mathematical structure in question." One reason the mapping account is unsatisfactory is that even if we accept it we can still ask why the existence of some appropriate mapping between the mathematical and the physical is sufficient to fully explain the particular application of the former to the latter. It is left unclear how a map from a mathematical structure to a physical one would illuminate how it is possible for features of the mathematical structure to play an indispensable role in explaining features of the physical one.

Another way the mapping account is deficient is that it leaves novel prediction from mathematical structure unexplained. A satisfactory solution to the applicability problem must explain how the following is possible: "In the case of novel predictions, by invoking suitable empirical interpretations of mathematical theories, scientists can draw inferences about the empirical world that the original scientific theory wasn't constructed to make" (Bueno and French 2012).

The mapping account cannot have anything to say about cases in which theorists accurately predict some future phenomenon that is not yet in existence. Suppose that scientists had predicted that hive-bee honeycomb would be hexagonally structured before any honeycombs actually existed. Biologists that knew of bees and their wax production could have predicted that hexagonally structured honeycomb would be an evolutionarily stable strategy and thus that bee populations that stumbled upon it and were stable over time would converge upon this strategy. How would the mapping account explain this? In this case, there would have been no actually instantiated empirical structure to which the relevant mathematical structure could be mapped, since the prediction would have been about an empirical phenomenon that did not yet exist (namely, hexagonally structured honeycomb). The relevant mapping would have to have been from the mathematical structure to the modal structure of the empirical system. The mapping could be between the mathematical structure and the equilibrium state, which is part of the modal structure. This already moves beyond the resources of Pincock's account. And there would still be the question of why this mathematical structure is applicable to the modal physical structure in such a way that it allows for novel prediction. This, again, is analogous to the parallel question for scientific realists of why theoretical entities explain and novelly predict observable phenomena.

In the case of scientific realism, we do not solve the no-miracles problem by merely positing theoretical entities. We must also spell out the relationship between the theoretical entities and the observable phenomena they were posited to explain. Ladyman and Ross (2007, p. 74) put it as follows: "It is only on the assumption that the unobservable entities posited by realists cause the phenomena that they explain them. If 
unobservable entities merely happened to be around when certain phenomena were occurring, then their presence would not be explanatory." In other words, in the context of the no-miracles argument it would still be miraculous if observable phenomena were explained by appeal to theoretical entities with which they were merely correlated rather than nomologically linked.

In cases of scientific explanation, the nomological relationship between unobservables and observables is often a causal one, though this is not true for every case. There are many examples in which unobservable entities and properties figure into non-causal explanations of observable phenomena. The Lorentz-invariance of spacetime, for instance, is not the sort of property to which we should ascribe causal powers, as it is a global symmetry of spacetime itself rather than a property instantiated at some location within spacetime. Yet, it plays an essential role in explaining why time dilation occurs. What is characteristic about all cases in which an unobservable property or entity explains an observable one is that there is some metaphysical-dependence relation between explanans and explanandum.

Once the constraint of metaphysical dependence is placed on the set of acceptable solutions to the applicability problem, the applicability of mathematics becomes less mysterious. One of the reasons we are able to make inferences from features about a certain mathematical structure to consequences about an empirical system is that the modal structure of the empirical system metaphysically depends on that mathematical structure. When we cannot make such inferences, one of the reasons may be that no such dependence relation holds between the structures.

\section{What kind of metaphysical dependence?}

I have argued that there must be some dependence relation between mathematical structure and modal physical structure. Facts about mathematical structures would not play an explanatory role in the empirical sciences if they "merely happened to be around." Facts about the modal properties of physical systems are grounded in facts about corresponding mathematical structures. Put another way, modal facts about physical systems hold in virtue of facts regarding their underlying mathematical structures. But how should we understand this in-virtue-of relation? What is the specific nature of the relationship between mathematical structure and the modal structure of the physical world? I address four possible relations that could do the work of grounding physical modality in mathematical structure. These are what I take to be prima facie the most promising options, though they are not exhaustive.

\subsection{Grounding}

If we invoke the relation put forth by Rosen (2010) and Schaffer (2015) we would understand the in-virtue-of relation that holds between modal physical structure and mathematical structure as in instance of the primitive big-G Grounding relation.

Grounding is a relation between facts or sets of facts, understood as true propositions. It is asymmetric and irreflexive. Rosen (2010) notes that we ought not assume the relation is a well-founded one. Since it is an open question as to whether there is a 
fundamental level of reality, it is epistemically possible that there is an infinite chain of facts such that each fact is grounded in a subsequent fact or set of facts. Grounding is said to be analogous with causation.

But it is not clear how much more can be said about Grounding. Wilson (2014) argues against Grounding on the basis that it is too course-grained to do the work required of it, it is not clearly one single thing over and above an aggregation of more specific small-g grounding relations, and it fails to be illuminating as a primitive relation.

These considerations against Grounding are not necessarily decisive. If it turns out to be a useful notion that does important metaphysical work, then perhaps it may be redeemed and put to work here. For our current purposes, however, exchanging the mystery of applicability for the no less mysterious metaphysical relation of Grounding is not a compelling solution.

\subsection{Supervenience}

On its face, supervenience may seem like a promising notion for understanding how modal physical structure depends on mathematical structure. However, it is a poorly suited candidate for our dependence relation. While supervenience is itself a modal notion, it cannot provide the necessary apparatus for grounding non-Humean modality in mathematical structure. Supervenience can be invoked to define a Humean picture of modal physical structure, where modality is grounded in patterns of regularity. What is key for supervenience to be able to do such work is that modality must be grounded in something that can change or that could have been different. On the Humean picture, modality is grounded in universal regularities, which themselves could be and could have been different.

On a picture where modality is grounded in features of mathematical structures, invoking supervenience means that the grounding relation never holds. Modal physical structure $\mathrm{P}$ supervenes on mathematical structure $\mathrm{M}$ just in case the following is true: There could have been no difference in $\mathrm{P}$ without a corresponding difference in $\mathrm{M}$. But if $\mathrm{M}$ is a mathematical structure there is no sense in which $\mathrm{M}$ could have been different, since mathematical structures have their mathematical features necessarily.

There is a sense in which the mathematical structure that underlies the modal structure of a given empirical system could have been different. But this is just what would be the case if a modal physical structure instantiates one mathematical structure but could have instantiated a different one. This leads us to our next option of understanding the grounding relation as one of instantiation.

\subsection{Instantiation}

Understanding modal physical structures as instantiating mathematical structures allows us to make sense of the thought that the modal structure of certain physical systems could have been different.

Consider the structure of spacetime. Suppose the modal structure of our physical spacetime instantiates the mathematical structure of a four-dimensional pseudo- 
Riemannian manifold with a Lorentz metric tensor. If our spacetime is a token of this mathematical type, it follows that our spacetime will have the symmetries that characterize the mathematical structure. From this, it can be derived that the empirical phenomenon of time dilation will arise.

Yet presumably our spacetime could have been different. If the modal physical structure of our spacetime had instantiated a toroid, for example, then different symmetries would be exhibited. Space would not have been isotropic. Apparently rigid bodies would appear to undergo expansion and contraction as they moved through space. The modal features of spacetime could have been different if spacetime had instantiated a different mathematical structure. ${ }^{12}$ Unlike supervenience, instantiation allows us to make sense of how the modal profile of spacetime could have been different.

The instantiation account also fills in what the mapping account leaves out. Why should the existence of a structural isomorphism between a mathematical structure and some aspect of the physical world allow us to make inferences about the physical world and predications about the future states of the physical system? On the instantiation account, the answer is that the mathematical structure constrains what future states of the system are possible. That the modal structure of the physical system instantiates the mathematical structure is what allows the mathematical structure to play the role of limiting and constraining the physical.

This sort of explanation is missing from the mapping account. Think about an actual map that corresponds to some area of the world. There has to be some explanation of how it came to be the case that the map strongly corresponds to the world. On this metaphor, however, it is the world that limits the map. The explanation for the correspondence would cite both the features of the world that were to be mapped out as well as whatever social or professional norms of cartography were that dictated that accurate representation of some salient aspects of the world were to be desired. On the mapping account, we understand the relationship between mathematical structure and the physical world to be one of mapping, and the world limits what mathematical structures can be used to successfully map its systems.

My suggestion is that, for the reasons given in Sect. 4, the mapping account gets the direction of the limiting relation the wrong way around. We ought to understand the mathematical structures that the modal structures of the physical world instantiate as limiting the possible features of the physical systems that instantiate them. On this view, we can understand our inferences from mathematical structures to modal facts as broadly in line with other inferences we make from features of a type to features of a token.

Instantiation provides the necessary premise that allows us to derive modal physical facts from mathematical ones. For instance, we know that the modal structure of the bridges of Königsberg problem instantiates a specific graph. It is a graph that has no Eulerian cycle and thus no trail that starts and ends at the same graph vertex. Now, the bridges of Königsberg have plenty of other contingent non-mathematical properties, but the physical property of being such that each bridge cannot be crossed exactly

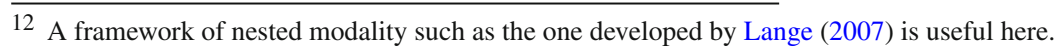


once derives from the corresponding mathematical property of having four vertices of odd degree and thus no Euler path.

The instantiation account gives us the resources to understand the fact that the mathematics limits possible features of physical systems, and it provides us with a template for understanding how inferences and derivations of the features of these systems from the mathematical structures they instantiate is possible.

\subsection{Identity}

Perhaps the strangest possibility is that mathematical structure and modal physical structure are simply one and the same. This could be thought of in one of two ways. Either the physical world is itself a mathematical structure or mathematical structures are just modal physical profiles. ${ }^{13}$ Tegmark (2007) defends a version of the former, while the latter fits with attempts to reduce the mathematical to modal possibilities about the physical.

The most obvious drawback of positing the identity relation is that, as discussed above, modal physical structures and mathematical structures seem to have different modal profiles. Seemingly the modal structure of an empirical system such as spacetime could have been different if the empirical system itself had been different. But the mathematical structures that underlie the modal structures could not have been different. So if we take modal properties to be real properties of these structures, it would seem that the modal and the mathematical could not be one and the same. There are likely ways to get around this difficulty, but they require additional work. ${ }^{14}$

\section{Concluding remarks}

This paper has explored a number of cases that demonstrate the indispensability of mathematics to the prediction and explanation of empirical phenomena. I have shown that the empirical phenomena in each case must be understood as inherently modal and that mathematics plays an essential role in explaining the modal features of these empirical systems. Why is mathematics able to predict and explain so much about the physical world? It is a lesson of scientific realism that, for theoretical entities to do explanatory work, they must bear some determination relation to the phenomena they are supposed to explain. I have argued that any solution to the applicability problem that the scientific realist can accept must appeal to a metaphysical dependence relation between mathematics and modal physical structure. While we might understand this metaphysical dependence in terms of any number of specific relations, the promise of the instantiation relation looms largest. Instantiation seems to be the candidate relation most able to accommodate the fact that some modal structures of physical systems

\footnotetext{
13 Of course identity is a two-way street. But when we talk about discovering that what we initially thought were two separate things, A and B, are actually one thing, this is frequently spelled out in terms of finding that A actually has all or most of the properties that we initially thought B had or vice versa.

14 Tegmark's picture of the mathematical multiverse on which all mathematical structures are physically realized somewhere in the multiverse may contain the resources to respond to this concern.
} 
could have been different, to shed light on how it is that we can derive facts about the physical world from features of mathematical structures, and to illuminate the way that mathematical facts limit what is possible in the physical world.

Acknowledgments Thank you to the following people for their helpful and patient discussions of this paper: Derek Anderson, Jordan Baker, Anjan Chakravartty, Kathleen Connelly, Josh Dever, David Frank, Steven French, Joyce Havstad, Lina Jansson, Cory Juhl, Ashley Kennedy, Gal Kober, Rob Koons, James Ladyman, Bryan Pickel, Katherine Ritchie, John Roberts, Juha Saatsi, Marlin Sommers, and Audrey Yap

\section{References}

Baker, A. (2009). Mathematical explanation in science. British Journal of Philosophy of Science, 60, 611633.

Baker, A. (2005). Are there genuine mathematical explanations of physical phenomena? Mind, 114(454), $223-238$.

Bangu, S. (2012). The applicability of mathematics in science: Indispensability and ontology. Basingstoke: Palgrave Macmillan.

Berenstain, N. (2014). Necessary laws and chemical kinds. Australasian Journal of Philosophy, 92(4), 631-647.

Berenstain, N. (2016). What a structuralist theory of properties could not be. In A. Marmodoro \& D. Yates (Eds.), The metaphysics of relations. Oxford: Oxford University Press.

Berenstain, N., \& Ladyman, J. (2012). Ontic structural realism and modality. In E. Landry \& D. Rickles (Eds.), Structural realism: Structure, object, and causality. New York: Springer.

Bueno, O., \& Colyvan, M. (2011). An inferential conception of the application of mathematics. Noûs, 45(2), 345-374.

Bueno, O., \& French, S. (2012). Can mathematics explain physical phenomena? British Journal for the Philosophy of Science, 63(1), 85-113.

Colyvan, M. (1999). Confirmation theory and indispensability. Philosophical Studies, 96(1), 1-19.

Colyvan, M. (2001a). The indispensability of mathematics. New York: Oxford University Press.

Colyvan, M. (2001b). The miracle of applied mathematics. Synthese, 127, 265-277.

Colyvan, M. (2006). Scientific realism and mathematical nominalism: A marriage made in hell. In C. Cheyne \& J. Worrall (Eds.), Rationality and reality: Conversations with Alan Musgrave (pp. 225237). Dordrecht: Springer.

Colyvan, M. (2010). There is no easy road to nominalism. Mind, 119(474), 285-306.

Goles, E., Schulz, O., \& Markus, M. (2001). Prime number selection of cycles in a predator-prey model. Complexity, 6(4), 33-38.

Hales, T. (2001). The honeycomb conjecture. Discrete and Computational Geometry, 25, 1-22.

Ladyman, J., Ross, D., Spurrett, D., \& Collier, J. (2007) Every thing must go.Metaphysics naturalised. Oxford: Oxford University Press.

Lange, M. (2007). Laws and meta-laws of nature: Conservation laws and symmetries. Studies in History and Philosophy of Science Part B: Studies in History and Philosophy of Modern Physics, 38(3), 457-481.

Leng, M. (2002). What's wrong with indispensability? (Or, the case for recreational mathematics). Synthese, 131(3), 395-417.

Lloyd, M., \& Dybas, H. S. (1966). The periodical cicada problem. II. Evolution. Evolution, 20, 466-505.

Lyon, A. (2012). Mathematical explanations of empirical facts, and mathematical realism. Australasian Journal of Philosophy, 90(3), 559-578.

Lyon, A., \& Colyvan, M. (2008). The explanatory power of phase spaces. Philosophia Mathematica, 16(2), $227-243$.

Melia, J. (2000). Weaseling away the indispensability argument. Mind, 109(435), 455-479.

Pincock, C. (2004a). A revealing flaw in colyvan's indispensability argument. Philosophy of Science, 71, 61-79.

Pincock, C. (2004b). A new perspective on the problem of applying mathematics. Philosophia Mathematica (3), 12, 135-161.

Pincock, C. (2007). A role for mathematics in the physical sciences. Noûs, 41, 253-275.

Psillos, S. (2002). Causation and explanation. New York: Routledge. 
Rizza, D. (2011). Magicicada, mathematical explanation and mathematical realism. Erkenntnis, 74(1), $101-114$.

Rosen, G. (2010). Metaphysical dependence: Grounding and reduction. In H. Bob \& A. Hoffmann (Eds.), Modality: Metaphysics, logic, and epistemology (pp. 36-109). Oxford: Oxford University Press.

Sober, E. (1983). Equilibrium explanation. Philosophical Studies, 43, 201-210.

Saatsi, J. (2011). The enhanced indispensability argument: Representational versus explanatory role of mathematics in science. British Journal for the Philosophy of Science, 62(1), 143-154.

Schaffer, J. (2015). Grounding in the image of causation. Philosophical Studies, 52, 1-1.

Steiner, M. (1978). Mathematics, explanation, and scientific knowledge. Nous, 12(1), 17-28.

Steiner, M. (1989). The application of mathematics to natural science. Journal of Philosophy, 86, 449-480.

Steiner, M. (1995). The applicabilities of mathematics. Philosophia Mathematica, 3, 129-156.

Steiner, M. (1998). The applicability of mathematics as a philosophical problem. Cambridge, MA: Harvard University Press.

Tegmark, M. (2007). The Mathematical Universe. arXiv:0704.0646

Weinberg, S. (1979). Conceptual foundations of the unified theory of weak and electromagnetic interactions. Nobel Lecture, December 8. Lyman Laboratory of Physics Harvard University and HarvardSmithsonian Center for Astrophysics Cambridge, MA, USA.

Wigner, E. P. (1960). The unreasonable effectiveness of mathematics in the natural sciences. Communications on Pure and Applied Mathematics, 13, 1-14.

Wilson, J. (2014). No work for a theory of grounding. Inquiry, 57(5-6), 535-579.

Ye, F. (2010). The applicability of mathematics as a scientific and a logical problem. Philosophia Mathematica, 18(2), 144-165. 